\title{
Design, Manufacturing and Tests of an Implantable Centrifugal Blood Pump
}

\author{
Eduardo Bock ${ }^{1,2,3}$, Pedro Antunes ${ }^{1,4}$, Beatriz Uebelhart ${ }^{1,5}$, Tarcísio Leão ${ }^{3}$, \\ Jeison Fonseca ${ }^{1,6}$, André Cavalheiro ${ }^{4}$, Diolino Santos Filho ${ }^{4}$, José Roberto Cardoso ${ }^{4}$, \\ Bruno Utiyama ${ }^{1}$, Juliana Leme ${ }^{1}$, Cibele Silva ${ }^{1}$, Aron Andrade ${ }^{1}$, and Celso Arruda ${ }^{2}$ \\ ${ }^{1}$ Institute Dante Pazzanese of Cardiology, IDPC \\ ${ }^{2}$ Unicamp, Campinas State University \\ ${ }^{3}$ Federal Institute of Technology, IFSP \\ ${ }^{4}$ Escola Politecnica of Sao Paulo University, EPUSP \\ ${ }^{5}$ Faculty of Technology, FATEC \\ ${ }^{6}$ Technological Institute of Aeronautics, ITA, Brazil \\ eduardo_bock@yahoo.com.br
}

\begin{abstract}
An implantable centrifugal blood pump was developed for long-term ventricular assistance in cardiac patients. In vitro tests were performed, as wear evaluation, performance tests and hemolysis tests in human blood. Numerical computational simulations were performed during design process in order to predict its best geometry. Wear evaluations helped to select the best materials for double pivot bearing system proposed to achieve longer durability. Performance tests pointed the best impeller geometry. The implantable centrifugal blood pump was compared with other blood pumps founded in literature. The proposed implantable centrifugal blood pump showed the best performance. But, its results showed a strong descendant curve in high flow. Other prototype was manufactured with a different inlet port angle to overcome this problem. The normalized index of hemolysis (NIH) measured $0.0054 \mathrm{mg} / 100 \mathrm{~L}$ that can be considered excellent since it is close to the minimum found in literature (between $0.004 \mathrm{~g} / 100 \mathrm{~L} \mathrm{e} 0.02 \mathrm{~g} / 100 \mathrm{~L}$ ). The authors' expectation is that this pump will become a promising Technological Innovation for Sustainability.
\end{abstract}

Keywords: Artificial Organs, Blood Pumps, Ventricular Assist Devices.

\section{Introduction}

A novel Implantable Centrifugal Blood Pump has been developed for long term circulatory assistance with a unique impeller design concept [1]. This feature was called dual impeller because it allies a spiral-shaped cone [2] with vanes to improve blood flow characteristics around the top inflow area to avoid blood clot due to stagnant flow (Fig. 1).

A series of previous studies demonstrated significant advantages from spiral shaped impeller design, providing axial characteristics to the flow in Left Ventricle Assist Devices [1]. The axial force component can avoid stagnant flow formation. Therefore, this principle can help to avoid thrombus related with blood stagnation [3]. 
This work presents results from Design, Manufacturing and Tests of an Implantable Centrifugal Blood Pump wear evaluation in double pivot bearing system, hydrodynamic performance tests with mock loop circuit, and preliminary normalized hemolysis tests.

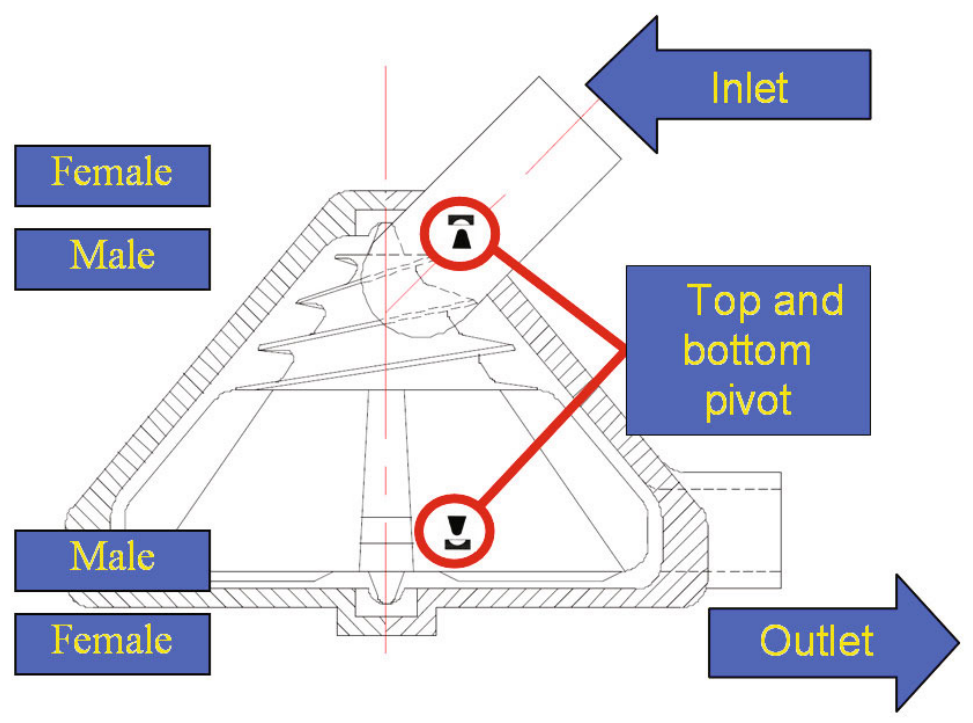

Fig. 1. The new Implantable Centrifugal Blood Pump for cardiac assistance is shown with its double pivot bearing system composed by male and female bearings

The double pivot bearing system has been used and studied in the last decade showing simplicity and reliability [4]. Recent studies evaluated the particle release from a centrifugal pump with double pivot bearing system composed of alumina ceramic (A12O3) and ultrahigh molecular weight polyethylene (UHMWPE). This pivot bearing system was tested under severe conditions showing very small risk of releasing debris particles to blood $[1,5]$.

\section{Contribution to Sustainability}

\subsection{The Design Process}

The design process was conducted according with current medical, social and financial needs of cardiac patients in Brazil. It is part of a multicenter and international study with objective to offer simple, affordable and reliable devices to developing countries since local health systems, both public and private health care, cannot afford the available technologies.

The authors have expectation that this pump will become a promising Technological Innovation for Sustainability in Brazil. Its simplicity is illustrated in (Fig. 2) showing two different options for motors and pump. 


\section{Motor A}
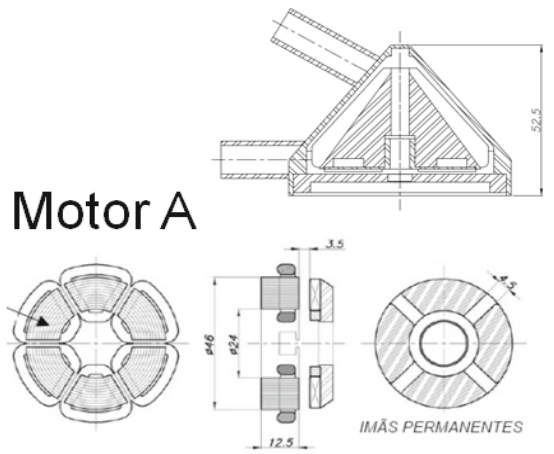

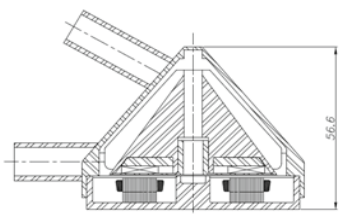

Motor B
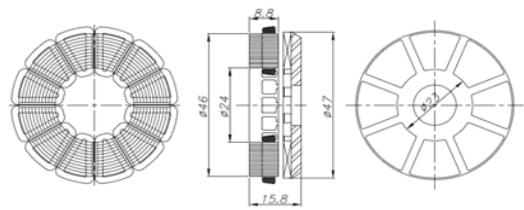

Fig. 2. The design process was lead according to current needs of cardiac patients who cannot afford Left Ventricular Assist Device technology. The reduced number of parts is shown in two different options of motor attached with pump's rotor by a magnetic coupling.

\subsection{Performance Tests}

Performance tests were conducted with several different prototypes, some of them machined in translucent materials and titanium, other made with Selective Laser Sintering (SLS) in Nylon and ABS polymer (Fig. 3 shows some pictures of prototypes).

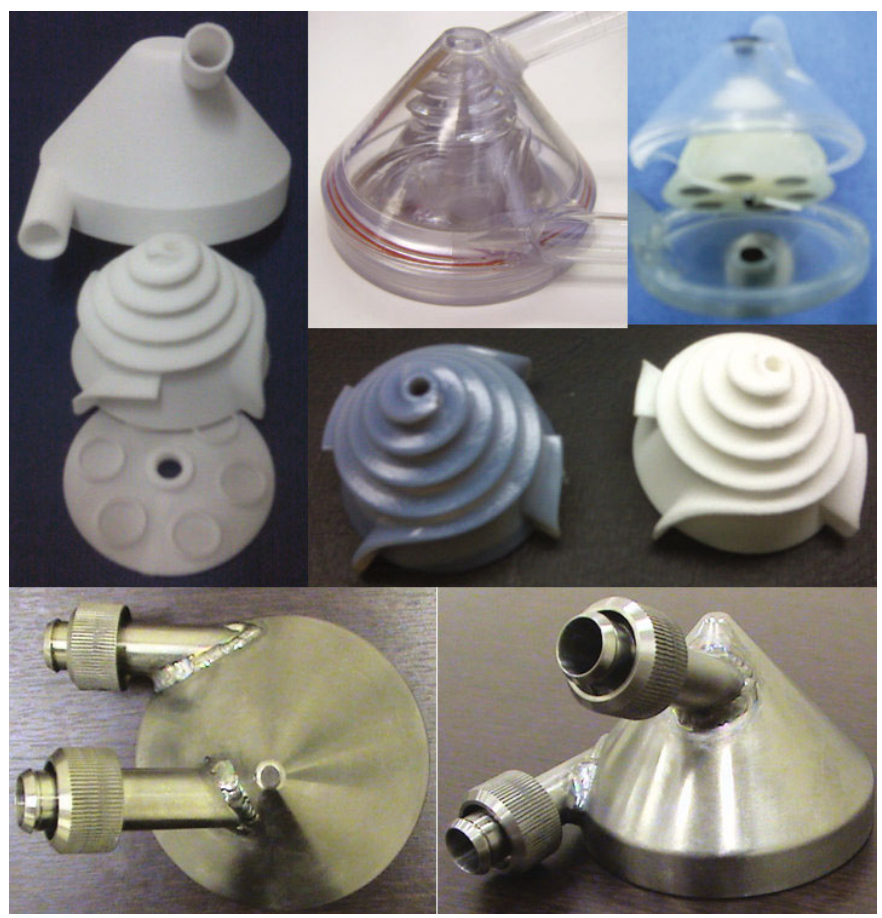

Fig. 3. Different types of prototypes obtained by SLS or Machined in different types of materials from translucent polymers to titanium 
In order to verify the viability of different materials, wear tests were made with several pivot bearings. Mainly, two types of defect were found, abrasive wear and surface fatigue. Previous tests with this system were performed with pumps working in mock loop circuits.

In order to study the wear phenomenon in each component of double pivot bearing, an isolated wear station was assembled to repeat this condition. In vitro performance tests were made in order to characterize hydraulic performance curves for the pump.

These tests can provide important information about the pump's capability and function ability. The generated curves can be used as a tool to predict which rotation is necessary to provide specific pressure and flow [6].

During performance tests, two different types of pump's inlet port were compared, with $45^{\circ}$ and $30^{\circ}$ of inlet angle. Finally, two isolated normalized tests of hemolysis with human blood were performed producing four values of normalized index of hemolysis (NIH), obtained from variation of plasma free hemoglobin (PFH), measured by a tetramethylbenzidine (TMB) assay method (Catachem, Bridgeport, CT, USA).

\section{Materials and Methods}

Wear Evaluation in Double Pivot Bearing System. A wear test station was assembled with the purpose of measuring isolated wear rate. It makes it possible to vary the shear stresses in consequence of charge applied to the system. The main idea was to evaluate wear using mass measurements and quantifying the bearing's material debris released in blood during the pump's operation [1].

Adapting a milling machine, the wear test station was assembled with rotation controller, water lubrication, depth controller, and applied charge measurement system. The wear tests were divided into three steps. In the first step, all bearings were weighted in a precision scale with divisions of $0.1 \mathrm{mg}$. After that, each pair of male and female bearing was tested. Two types of pairs were tested, ceramic-polymeric pairs and ceramic-ceramic pairs. After the tests, the pairs were weighted again to measure the wear loss in mass.

The polymers chosen were nylon, UHMWPE, and Teflon (poly-tetrafluorethylene).The ceramics chosen were zirconium dioxide $(\mathrm{ZrO} 2)$, silicon nitride (Si3N4), carbon, and alumina (Al2O3).

All pieces tested had their contact surfaces polished and roughness controlled to assure an average of 0.1 to $0.5 \mathrm{~mm}$. Each test was performed under the following conditions: room temperature $21^{\circ} \mathrm{C}, 9.8 \mathrm{~N}$ of charge applied at $4000 \mathrm{rpm}$ for a total of 40000 revolutions.

Hydrodynamic Performance Tests with Mock Loop Circuit. A performance test circuit was assembled with one hanging flexible reservoir sac (Flexible Sac, 3M, St. Paul, Minnesota, USA), two pressure probes, one pressure monitor, one ultrasonic flowmeter with 3/8" probe (Transonic Systems, Ithaca, NY, USA), pump and controller, acquisition PCI slot, and a laptop with Labview software (National Instruments, Austin, TX, USA).

The mock loop circuit was set with water solution with $37 \%$ of volume filled with glycerin to simulate the blood viscosity and density. The hanging flexible reservoir was filled with $0.4 \mathrm{~L}$ and placed $0.5 \mathrm{~m}$ above the pump. The pressure gauges were 
connected $0.3 \mathrm{~m}$ from pump inlet and outlet ports. The ultrasonic flowmeter transducer was located $0.15 \mathrm{~m}$ from pump outlet. Total length of each tubing from pump inlet and outlet to reservoir sac was $1.0 \mathrm{~m}$, Fig. 4 . The mock circuit was set horizontal to the table for easy adjustment of pump afterload using a screw clamp. Any air was removed from the circuit before the data acquisition [7].
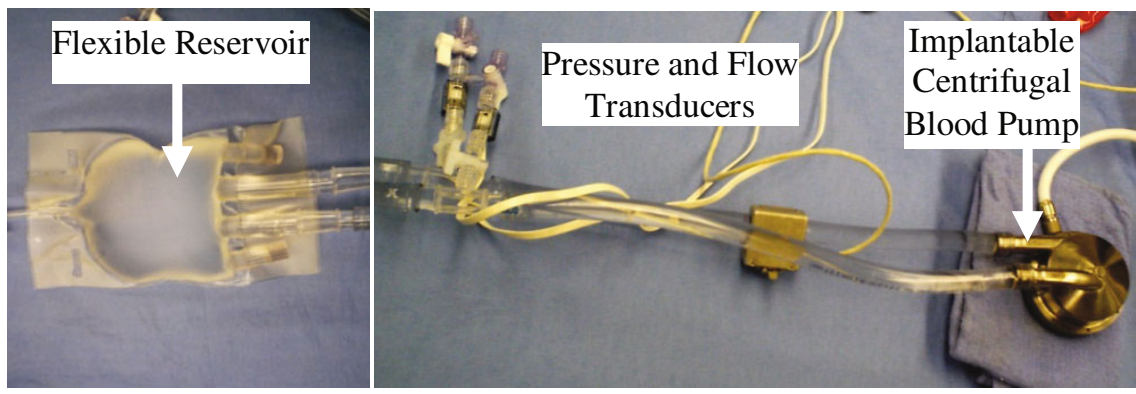

Fig. 4. Simple mock loop circuit assembled for data acquisition during performance tests

Two pumps were tested with different inlet angles, $45^{\circ}$ and $30^{\circ}$. The angles were chosen according to similar devices. The pump speed rotations were fixed at 1200 , $1400,1600,1800,2000$, and $2200 \mathrm{rpm}$. The software plotted a curve for each rotation, and for each pump. With screw clamp open, the first point collected was the maximum flow for each pump speed. Closing the screw clamp, the pump afterload increases as the flow decreases, and the next points are collected each $0.5 \mathrm{~L} / \mathrm{min}$, successively until $0.0 \mathrm{~L} / \mathrm{min}$ is achieved, when the clamp is totally closed.

The pump speed rotations were fixed at 1200, 1400, 1600, 1800, 2000, and 2200 $\mathrm{rpm}$. The software plotted a curve for each rotation, and for each pump. With screw clamp open, the first point collected was the maximum flow for each pump speed. Closing the screw clamp, the pump afterload increases as the flow decreases, and the next points are collected each $0.5 \mathrm{~L} / \mathrm{min}$, successively until $0.0 \mathrm{~L} / \mathrm{min}$ is achieved, when the clamp is totally closed.

Normalized Index of Hemolysis (NIH) Tests. According to ASTM Standard Practices F1830 and F1841, the hemolysis test was divided into five steps: collection and preparation of blood, mock loop setup, $6 \mathrm{~h}$ test, PFH measurement, and NIH calculation.

Blood was drawn from human volunteer using a large bore needle into a $500 \mathrm{~mL}$ blood bag with heparin to avoid clot formation during all procedures. No negative pressure exceeded $100 \mathrm{~mm} \mathrm{Hg}$ and the blood temperature was controlled between $2^{\circ} \mathrm{C}$ to $8^{\circ} \mathrm{C}$.

A test loop was assembled with $2 \mathrm{~m}$ of flexible $3 / 8^{\prime \prime}$ polyvinyl chloride tubing, a flexible reservoir with sampling port, an ultrasonic flowmeter with probe, a pressure monitor with probes, a thermistor with thermometer, and the blood pump (similar to performance station shown in Fig. 4). Blood had the hematocrit range between $28 \%$ to $32 \%$ controlled by hemodilution with phosphate-buffered saline.

Temperature was adjusted to $37^{\circ} \mathrm{C}$. The pump rotation was adjusted to provide $5 \mathrm{~L} / \mathrm{min}$ flow rate. A screw clamp was set around flexible tubing at the pump outlet 
side to produce the required pressure conditions for left ventricle assist devices (100 $\mathrm{mm} \mathrm{Hg}$ ). In each $6 \mathrm{~h}$ test, seven samples were collected T0,T1,T2,T3,T4,T5, and T6, and their respective PFH was measured.

\section{Results}

Ranking. The results of wear evaluation in different materials tested were sorted by loss of mass, and a ranking list of 10 best results was plotted in Figure 5. Values express the total loss of mass caused by wear in pairs and its total weight, with standard deviation of 0,000022 for measures.

Performance curves. A diagram (Flow vs. Pressure Ahead, as shown in Figure 6) was plotted with each pump curve superimposed in order to better understand the differences between both hydrodynamic performances.

\begin{tabular}{lllc}
\hline & Male bearing & Female bearing & Wear loss $(\mathrm{g})$ \\
\hline 1 st & Alumina & UHMWPE & 0.0001 \\
& Silicon nitride & Silicon nitride & 0.0001 \\
& Carbon & UHMWPE & 0.0001 \\
\multirow{5}{*}{5 th } & Carbon & Nylon & 0.0001 \\
& Alumina & PTFE & 0.0002 \\
& Silicon nitride & Nylon & 0.0002 \\
8th & Carbon & PTFE & 0.0002 \\
& Alumina & Nylon & 0.0003 \\
10 th & Zirconium dioxide & UHMWPE & 0.0003 \\
& Zirconium dioxide & Nylon & 0.0006 \\
\hline
\end{tabular}

UHMWPE, ultrahigh molecular weight polyethylene; PTFE, poly-tetra-fluorethylene (Teflon).

Fig. 5. Ranking of materials under wear tests

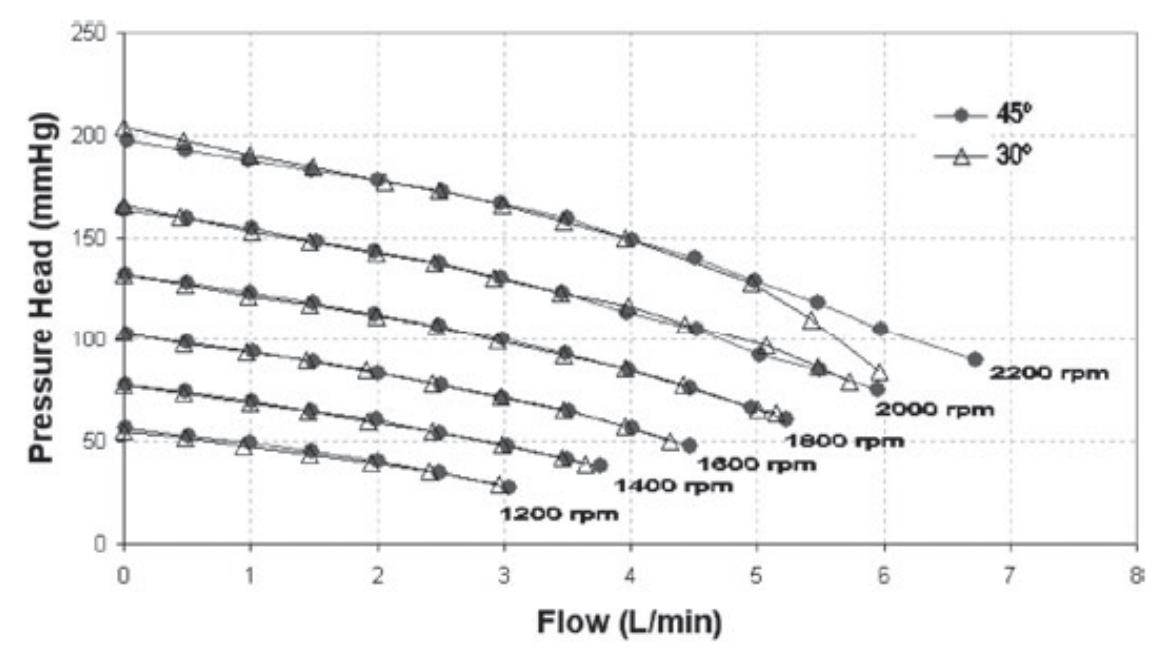

Fig. 6. Performance curves showing hydrodynamic characteristics for both pumps 
PFH releasing. PFH value was measured for each sample. With this PFH variation in time $\mathrm{T} 0, \mathrm{~T} 1, \mathrm{~T} 2, \mathrm{~T} 3, \mathrm{~T} 4, \mathrm{~T} 5$, and $\mathrm{T} 6$ is possible to represent hemoglobin releasing in plasma during the experiment. This progressive alteration in PFH levels in blood is caused by trauma imposed by pump.

\section{Discussion}

As described in previous studies [1], the bearing composed of alumina with UHMWPE showed the best results in wear evaluation trials with ceramic-polymeric pairs [8]. Four pairs had the minimum mass loss $(0.1 \mathrm{mg})$ : alumina with UHMWPE, silicon nitride with silicon nitride, carbon with UHMWPE, and carbon with nylon.

Pivot bearing systems composed only of ceramic are known to have higher vibration during pumping applications instead of shock absorption experienced in ceramicpolymeric pivot bearing systems $[4,7]$.

The pump with inlet angle of $45^{\circ}$ showed best performance results compared with other pumps built with inlet angle of $30^{\circ}$. Slight differences among curves were found in rotations beyond $1800 \mathrm{rpm}$ when the curves from $30^{\circ}$ pump decrease pressure ahead versus flow. Inlet port's angle is a problem to deal with when designing centrifugal blood pumps, especially the eccentric inlet port $[9,10]$.

As described by several authors, NIH for LVAD should be between 0.004 to 0.02 $\mathrm{mg} / 100 \mathrm{~L}$ to be considered satisfactory and antitraumatic blood pump $[1,6,10]$. After calculations, the preliminary hemolysis tests showed an NIH value of 0.0054 $\pm 2.46 \times 10^{-3} \mathrm{mg} / 100 \mathrm{~L}$.

\section{Conclusions}

The pair composed of alumina and UHMWPE was chosen to be the materials of the double pivot bearing system in order to avoid vibration problems.

The dual impeller centrifugal blood pump had proven to be a promising LVAD. The hydraulic characteristics are similar to other reported curves from established, durable, and reliable devices.

The authors' expectation is that this pump will become a promising Technological Innovation for Sustainability.

\section{Acknowledgments}

The authors are grateful to Dr. Yukihiko Nosé for enormous collaboration to this project. Also, it is mandatory to thank all colleagues from Baylor College of Medicine (BCM), Federal Institute of Technology (IFSP) as well as CNPq, HCor and Adib Jatene Foundation (FAJ) for partially supporting this research.

\section{References}

1. Bock, E., Antunes, P., Andrade, A., et al.: New Centrifugal Blood PumpWith Dual Impeller and Double Pivot Bearing System:Wear Evaluation in Bearing System, Performance Tests, and Preliminary Hemolysis Tests. Artif. Organs 32, 329-333 (2008) 
2. Andrade, A., Biscegli, J., Dinkhuysen, J., et al.: Characteristics of a blood pump combining the centrifugal and axial pump principles. Artif. Organs 20, 605-612 (1996)

3. Yamane, T., Miyamoto, Y., Tajima, K., et al.: A comparative study between flow visualization and computational fluid dynamic analysis for the sun medical centrifugal blood pump. Artif. Organs 28, 458-466 (2004)

4. Ohara, Y., Sakuma, I., Makinouchi, K., et al.: Baylor Gyro pump: a completely seal-less centrifugal pump aiming for long-term circulatory support. Artif. Organs 17, 599-604 (1993)

5. Takami, Y.: In vitro study to estimate particle release from a centrifugal blood pump. Artif. Organs 30, 371-376 (2006)

6. Takami, Y., Andrade, A., Nosé, Y., et al.: Eccentric inlet port of the pivot bearing supported Gyro centrifugal pump. Artif. Organs 21, 312-317 (1997)

7. Hansen, E., Bock, E., Nosé, Y., et al.: Miniaturized all-in-one rpm controllable actuator for gyro centrifugal blood pump. In: 52nd ASAIO Annual Conference, Chicago (2006)

8. Takami, Y., Nakazawa, T., Makinouchi, K., et al.: Material of the double pivot bearing system in the Gyro C1E3 centrifugal pump. Artif. Organs 21, 143-147 (1997)

9. Andrade, A., Biscegli, J., Souza, J., et al.: Flow visualization studies to improve the spiral pump design. Artif. Organs 21, 680-685 (1997)

10. Nosé, Y.: Design and development strategy for the rotary blood pump. Artif. Organs 22, 438-446 (1998) 